BULLETIN OF THE

AMERICAN MATHEMATICAL SOCIETY

Volume 77, Number 3, May 1971

\title{
ORIENTATION-PRESERVING MAPPINGS, A SEMIGROUP OF GEOMETRIC TRANSFORMATIONS AND A CLASS OF INTEGRAL OPERATORS ${ }^{1}$
}

\author{
BY ANTONIO O. FARIAS
}

Communicated by H. S. M. Coxeter, September 29, 1970

Let $A, B$ be smooth $\left(=C^{\infty}\right)$, oriented $n$-manifolds, $A$ with naturally oriented boundary, $\partial A$, and $B$ without boundary.

A very important problem in geometric analysis is that of giving an algebraic and/or combinatorial characterization of those smooth mappings from $\partial A$ to $B$ which can be extended to a smooth, orientation-preserving mapping from $A$ to $B$.

In this work, one such characterization is given in the particular case where $A$ is the unit disk, $D\left(\partial D=S^{1}\right)$, and $B$ is the plane, $R^{2}$. An application is made to a class of convolution-type operators to show they are topologically equivalent to the Hilbert transform.

1. Preliminaries. A smooth $f: S^{1} \rightarrow R^{2}$ is called extendable if there is a smooth $F: D^{-} \rightarrow R^{2}\left(D^{-}\right.$closure of $\left.D\right)$ with nonnegative Jacobian, $J_{F}$, and whose restriction to $S^{1}$ is $f$. If, further, $J_{F}>0$ on $S^{1}$ then $f$ is properly extendable.

A Titus transformation $T$ is a linear operator on the vector space of smooth functions from $S^{1}$ to $R^{2}$ given by:

$$
(T f)(t)=f(t)+c(t) \operatorname{det}\left[v, f^{\prime}(t)\right] v,
$$

$c$ a nonnegative, smooth function on $S^{1}$. The set of all finite compositions of Titus transformations is a semigroup, 3 . The effect of a Titus transformation can be represented by an elementary operation of growth along a fixed direction, growth understood in the sense of moving to the outside of an oriented curve.

A "degenerate" mapping $f: S^{1} \rightarrow R^{2}$ is one whose image lies in a onedimensional subspace. A Titus mapping ( $T$-mapping) is the image by an element of $\zeta$ of a degenerate mapping. A Titus mapping, thus, has

AMS 1970 subject classifications. Primary 57D40, 47D05, 44A35; Secondary 30A90, 47E05, 44A15.

Key words and phrases. Normal immersions, extendable mappings, holomorphic mappings, Hilbert transform.

1 This research is contained in the author's doctoral dissertation submitted to the University of Michigan, and was supported by a Scholarship from Conselho Nacional de Pesquisas (Brazil). The author wishes to thank his advisor, Professor Charles Titus, for suggesting the problem and for his assistance throughout the work. 
a simple, basic, geometric meaning: it is a mapping which can be obtained as a finite number of growth operations applied to a degenerate curve.

\section{Results.}

Theorem 1. A normal mapping (see [4] for definition) is extendable if and only if it is a Titus mapping.

The if part is proved by induction and is due to C. J. Titus (oral communication). The only if part follows from Theorem 2 below.

TheOREM 2. Every properly extendable mapping is a Titus mapping.

The proof of Theorem 2 is accomplished by reduction to the case of a holomorphic function having only a simple zero of the derivative, in which case a direct construction is performed.

3. Application. Consider integral operators given by:

$$
y(t)=-\int_{0}^{2 \pi} k(s) x(t-s) d s,
$$

where $x$ is smooth and has period $2 \pi$, and $k$ is real analytic in $(0,2 \pi)$ with

$$
k^{\prime}(s)=\int_{-\infty}^{\infty} e^{-s r} d \mu(r),
$$

$\mu$ a nondecreasing function (see [1], [2]). We allow certain cases where $k$ is not integrable; the integral in (2.1) must, then, be interpreted in the sense of Cauchy's principal value. (See [2] for details.)

Such an operator will be called a BL-operator. A mapping $f: S^{1} \rightarrow R^{2}$ is called a $B L$-mapping if it can be expressed as $f(t)=x(t)+i y(t)$ where $x, y$ satisfy (2.1) and (2.2).

It is easy to see that a holomorphic boundary [3] is a $B L$-mapping. We prove that they are generic, in some sense.

THEOREM 3. Every normal BL-mapping is topologically equivalent to a holomorphic boundary.

The proof uses approximation by $T$-mappings and results of Stoïlow-Whyburn-Carathéodory [3] about extendable mappings.

Full details will be published elsewhere.

\section{REFERENCES}

1. D. C. Benson, Extensions of a theorem of Loewner on integral operators, Pacific J. Math. 9 (1959), 365-377. MR 21 \#7406. 
2. C. Loewner, $A$ topological characterization of a class of integral operators, Ann. of Math. (2) 49 (1948), 316-332. MR 9, 502.

3. C. J. Titus, The combinatorial topology of analytic functions on the boundary of the disk, Acta Math. 106 (1961), 45-64.

4. H. Whitney, On regular closed curves in the plane, Compositio Math. 4 (1937), 276-284.

Eastern Michigan University, Ypsilanti, Michigan 48197 